\title{
Plantas de ocorrência espontânea como substratos alternativos para fitoseídeos (Acari, Phytoseiidae) em cultivos de seringueira Hevea brasiliensis Muell. Arg. (Euphorbiaceae)
}

\author{
Marcos Roberto Bellini ${ }^{1}$, Gilberto José de Moraes $^{2}$ \& Reinaldo José Fazzio Feres ${ }^{3}$ \\ 1 Programa de Pós-graduação em Entomologia Agrícola, Universidade Estadual Paulista. Via de acesso Professor Paulo \\ Donato Castellane, 14884-900 Jaboticabal, São Paulo, Brasil. Bolsista da FAPESP. E-mail: mrbellini@yahoo.com.br \\ 2, Departamento de Entomologia, Fitopatologia e Zoologia Agrícola, ESALQ/USP. Caixa Postal 9, 13418-900 Piracicaba, São \\ Paulo, Brasil. Pesquisador do CNPq. E-mail: gjmoraes@carpa.ciagri.usp.br \\ ${ }^{3}$ Departamento de Zoologia e Botânica, Universidade Estadual Paulista. Rua Cristóvão Colombo 2265, Jardim Nazareth, \\ 15054-000 São José do Rio Preto, São Paulo, Brasil. E-mail: reinaldo@dzb.ibilce.unesp.br
}

\begin{abstract}
Weeds as alternative substrates to phytoseiids (Acari, Phytoseiidae) in rubber tree Hevea brasilienis, Muell.Arg. (Euphorbiaceae) cultivation. The objective of this study was to determine weed species of rubber tree cultivation areas that could serve as reservoirs of predatory mites. The work was conducted in Olímpia, State of São Paulo, Brazil, in two rubber tree plantations. Every three months, about $1.000 \mathrm{~cm}^{3}$ of leaves of each one of the five dominant weeds in each plantation was taken to determine the phytoseiids. As the dominant weeds varied during the study, a total of 20 species were evaluated. Only Cecropia sp. was a dominant weed throughout the study, in both plantations. A total of 336 phytoseiids of the following species were found: Euseius citrifolius Denmark \& Muma, 1970, E. concordis (Chant, 1959), Galendromus annectens (DeLeon, 1958), Iphiseiodes zuluagai Denmark \& Muma, 1972, Neoseiulus anonymus (Chant \& Baker, 1965) and N. tunus (DeLeon, 1967). E. citrifolius (189 specimens) and N. tunus (138 specimens) were the most abundant species. The highest abundance ( 231 specimens) and diversity ( 5 species) were observed on Cecropia sp. On this plant was found the largest number of mites per sample (29 specimens), followed by Piper duncum Linnaeus (22), Guarea sp. (18) and Ageratum conyzoides Linnaeus (12). E. citrifolius has been determined as the most abundant predatory mite on rubber trees in the region where this work was conducted. Cecropia sp. seems to be the most important of the weeds considered as reservoir of E. citrifolius because it is continuously present in the plantations. Complementary studies could indicate the viability of managing this weed in or around rubber tree plantations to promote the biological control of mite pests on that crop.
\end{abstract}

KEY WORDS. Biological control, ecological management, pest management.

RESUMO. O objetivo deste estudo foi determinar as espécies de plantas espontâneas em áreas de cultivo de seringueira que poderiam servir com reservatório de ácaros predadores. O trabalho foi conduzido em Olímpia, Estado de São Paulo, Brasil, em dois cultivos de seringueira. A cada três meses, cerca de $1.000 \mathrm{~cm}^{3}$ de folhas de cada uma das cinco espécies de plantas espontâneas dominantes em cada cultivo foi tomada para determinar os fitoseídeos. Como as plantas espontâneas dominantes variaram durante o estudo, um total de 20 espécies foi verificado. Somente Cecropia sp. foi dominante em todo estudo, em ambos os cultivos. Um total de 336 fitoseídeos pertencentes às seguintes espécies foi encontrado: Euseius citrifolius Denmark \& Muma, 1970, E. concordis (Chant, 1959), Galendromus annectens (DeLeon, 1958), Iphiseiodes zuluagai Denmark \& Muma, 1972, Neoseiulus anonymus (Chant \& Baker, 1965) e N. tunus (DeLeon,1967). E. citrifolius (189 espécimes) e N. tunus (138 espécimes) foram os mais abundantes. A maior abundância (231espécimes) e diversidade (5 espécies) foram observadas sobre Cecropia sp. Sobre esta planta foi encontrado o maior número de ácaros por amostra (29 espécimes), seguida por Piper duncum Linnaeus (22), Guarea sp. (18) e Ageratum conyzoides Linnaeus (12). E. citrifolius tem sido determinado como um dos ácaros predadores mais abundantes sobre seringueiras cultivadas na região onde este trabalho foi conduzido. Cecropia sp. parece ser uma das plantas espontâneas mais importantes consideradas como reservatório de E. citrifolius porque está continuamente presente nos cultivos. Estudos complementares poderiam indicar a viabilidade de se manejar esta planta nas áreas de cultivo de seringueira para permitir o controle biológico dos ácaros-praga desta cultura.

PALAVRAS CHAVE. Controle biológico, manejo de praga, manejo ecológico.

Revista Brasileira de Zoologia 22 (1): 35-42, março 2005 
Nativa da região amazônica, a seringueira Hevea brasiliensis Muell. Arg. (Euphorbiaceae) foi introduzida em vários estados brasileiros, destacando-se a região noroeste do Estado de São Paulo. Nesta região, diversas espécies de ácaros têm sido encontradas associadas a esta planta (Feres 2000). Duas espécies vêm merecendo grande destaque: Calacarus heveae Feres, 1992, que pode causar severo desfolhamento e amarelecimento em diversos clones de seringueiras (VIeira \& Gomes 1999, Feres 2000), e Tenuipalpus heveae Baker, 1945, que pode causar considerável bronzeamento seguido de queda das folhas (PonTier et al. 2001).

As chamadas plantas espontâneas, também conhecidas como plantas invasoras ou daninhas, ocorrem naturalmente e têm crescimento espontâneo em áreas de cultivo. Experimentos de campo mostram que a diversificação cuidadosa e adequada da vegetação de plantas espontâneas em agroecossistemas pode diminuir de maneira significativa as populações de espécies pragas (Altieri et al. 2003). Uma das explicações para esse fato seria uma maior diversidade e disponibilidade de recursos alternativos (pólen, néctar, presas alternativas, entre outros) para os inimigos naturais das pragas (AlTiERI et al. 2003). Ácaros predadores da família Phytoseiidae receberam muita atenção a partir dos anos 50, quando se tornou evidente que algumas espécies eram importantes inimigos naturais de ácaros da família Tetranychidae em agroecossistemas (Moraes et al. 2004, Gerson et al. 2003). O estudo de ácaros predadores em plantas nativas é de fundamental importância, assumindo que estes possam naturalmente migrar destas às plantas cultivadas. Saber quais plantas são mais comumente encontradas associadas às culturas, assim como quais são os predadores nelas encontrados pode facilitar possíveis estratégias de manejo.

Ageratum conyzoides Linnaeus (Asteraceae) é uma das plantas espontâneas que tem sido estudada como reservatório de ácaros predadores no Estado de São Paulo. Gravena et al. (1993) avaliaram o efeito da semeadura de A. conyzoides e Eupatorium pauciflorum Kunth (Asteraceae) sobre a densidade de ácaros predadores e fitófagos em uma área experimental de citros em Jaboticabal, Estado de São Paulo. Os autores constataram uma maior abundância de Iphiseiodes zuluagai e Euseius citrifolius nas folhas de laranja na presença das plantas espontâneas. A incidência do ácaro da falsa ferrugem Phyllocoptruta oleivora (Ashmead, 1879) e do ácaro da leprose Brevipalpus phoenicis (Geijskes, 1939) também foi menor na presença destas plantas herbáceas. Trabalhos com a mesma planta em outros países também mostram a importância desta como reservatório de predadores, principalmente fitoseídeos (MAI et al. 1984?, LIANG et al. 1994, Mele \& Lenteren 2002). Em relação à seringueira, Feres \& Nunes (2001) estudaram três espécies de euforbiáceas herbáceas associadas ao cultivo e relataram a presença de sete espécies de fitoseídeos. ZaCARIAS \& Moraes (2001) realizaram um levantamento da população de predadores associados à seringueira e outras 11 euforbiáceas nativas no Sudeste do Estado de São Paulo, assinalando 25 espécies de fitoseídeos.

Além de funcionar como reservatórios de ácaros preda- dores, algumas plantas também podem funcionar como reservatórios de pragas. EMDEN (1965) citou 442 referências relacionando as plantas espontâneas como reservatório de pragas. THResh (1981) citou exemplos de patógenos de plantas que também podem ser encontrados em plantas espontâneas. Assim, não basta saber apenas se as plantas espontâneas podem abrigar predadores com potencial para o controle biológico, mas também se estas plantas podem servir de reservatórios de pragas e patógenos. Freqüentemente, muitos dos ácaros fitófagos encontrados sobre as plantas espontâneas não causam qualquer dano à cultura principal, podendo servir como alimento aos ácaros predadores encontrados sobre aquelas plantas.

Plantas consideradas "espontâneas" são comumente encontradas em plantios de seringueira, sendo capazes de se dispersar e se estabelecer nas entrelinhas do seringal (Lam et al. 1993, Ismail et al. 1995). Para subsidiar futuros estudos de controle biológico de ácaros danosos à seringueira, o presente trabalho teve como objetivo conhecer as espécies de ácaros fitoseídeos que ocorrem em plantas espontâneas abundantes em cultivos de seringueira, para verificar quais daquelas poderiam servir como reservatórios de fitoseídeos, no noroeste do Estado de São Paulo.

\section{MATERIAL E MÉTODOS}

Duas áreas de cultivo de seringueira no município de Olímpia foram investigadas, sendo uma delas consorciada com gariroba, Syagrus oleracea (Mart.) Becc. (Arecaceae). Estas são referidas no presente trabalho como área I $\left(20^{\circ} 43^{\prime} 53^{\prime \prime} \mathrm{S}, 4^{\circ} 03^{\prime}\right.$ $\left.38^{\prime \prime} \mathrm{W}\right)$ e área II $\left(20^{\circ} 42^{\prime} 59^{\prime \prime} \mathrm{S}, 49^{\circ} 01^{\prime} 58^{\prime \prime} \mathrm{W}\right)$, respectivamente. As amostragens foram realizadas trimestralmente, no meio de cada estação (julho e outubro de 2002 e janeiro e abril de 2003). Em cada dia de amostragem, as cinco espécies de plantas espontâneas visualmente dominantes em cada área foram determinadas. Tomaram-se, então, três amostras de folhas de plantas de cada espécie vegetal de diferentes partes do seringal, cada uma correspondendo a um volume de aproximadamente $1000 \mathrm{~cm}^{3}$. Cada amostra foi acondicionada em um saco de papel, que por sua vez foi colocado em um saco de polietileno. Os sacos foram colocados em caixas de poliestireno em que a temperatura foi mantida abaixo de $20^{\circ} \mathrm{C}$ pela adição de unidades plásticas contendo um gel previamente congelado $\left(\right.$ Gelo- $\left.\mathrm{X}^{\odot}\right)$, para transporte ao laboratório. As amostras foram guardadas em refrigerador $\left(\cong 10^{\circ} \mathrm{C}\right.$ ) por um período máximo de sete dias, até que fossem examinadas sob estereomicroscópio para a coleta dos fitoseídeos.

Os fitoseídeos encontrados foram montados em meio de Hoyer (Flechtmann 1975) e identificados com base nas descrições e redescrições de cada espécie.

\section{RESULTADOS}

Foram amostradas 12 espécies de plantas em cada área de cultivo (Tab. I). Como somente quatro espécies foram amostradas em ambas as áreas, 20 espécies de plantas foram amostra- 
das no total. Somente Cecropia sp. (Cecropiaceae) foi amostrada em todas as coletas, pois foi a única espécie de planta que esteve entre as cinco dominantes em todas as coletas nas duas áreas de cultivo. Cayaponia sp. (Cucurbitaceae) foi uma das dominantes em duas ocasiões em cada uma das áreas. Piper sp. (Piperaceae) foi uma das dominantes em três ocasiões na área I e em uma ocasião na área II. Panicum maximum Jacq. (Poaceae) foi uma das dominantes em três ocasiões na área II. As demais foram dominantes somente uma ou duas vezes em cada área de cultivo.

Foram coletados 336 espécimes de fitoseídeos distribuídos em seis espécies:

\section{Euseius citrifolius Denmark \& Muma, 1970}

Euseius citrifolius Denmark \& Muma, 1970: 222; Moraes \& McMurtry, 1983: 138; Noronha \& Moraes, 2002: 1114.

Plantas em que foi encontrado: A. conyzoides, Amphilophium sp., Astronium sp., Capsicum sp., Cecropia sp., Erechtites sp., Eupatorium sp., Guarea sp., Heliotropium sp., Lantana sp., P. maximum, Piper aduncum Linnaeus, Piper sp., Solanum americanum Mill. e Sonchus sp.

\section{Euseius concordis (Chant, 1959)}

Typhlodromus (Amblyseius) concordis Chant, 1959: 69. Amblyseius (Iphiseius) concordis Muma, 1961: 288. Amblyseius concordis Chant \& Baker, 1965: 22.

Euseius concordis Denmark \& Muma, 1973: 264; Moraes \& McMurtry, 1983: 138; Noronha \& Moraes, 2002: 1116.

Euseius flechtmanni Denmark \& Muma, 1970: 223; Denmark \& Muma, 1973: 261 (sinonímia de acordo com Moraes et al., 1982: 18)

Plantas em que foi encontrado: A. conyzoides e Lantana sp.

\section{Galendromus annectens (DeLeon, 1958)}

Typhlodromus annectens DeLeon, 1958: 75; Chant \& YoshidaShaul, 1984: 1868; Moraes \& McMurtry, 1983: 142; Moraes \& Mesa, 1988: 82; Zacarias \& Moraes, 2001: 583.

Galendromus annectens Muma, 1961: 298; Muma, 1963: 20; Denmark \& Muma, 1973: 274; Moraes et al., 1982: 21.

Planta em que foi encontrado: Cecropia sp.

\section{Iphiseiodes zuluagai Denmark \& Muma, 1972}

Iphiseiodes zuluagai Denmark \& Muma, 1972: 23; Denmark \& Muma, 1973: 251; Moraes et al., 1982: 18; Aponte \& McMurtry, 1995: 176.

Amblyseius zuluagai Moraes \& Mesa, 1988: 79.

Plantas em que foi encontrado: Cecropia sp. e Lantana sp.

\section{Neoseiulus anonymus (Chant \& Baker, 1965)}

Amblyseius anonymus Chant \& Baker, 1965: 21; Schicha \& Elshafie, 1980: 32; McMurtry, 1983: 254.
Neoseiulus anonymus Denmark \& Muma, 1973: 265; Moraes \& Mesa, 1988: 76; Moraes et al., 2000: 245.

Plantas em que foi encontrado: Cecropia sp.

\section{Neoseiulus tunus (DeLeon, 1967)}

Typhlodromips tunus DeLeon, 1967: 29; Denmark \& Muma, 1973: 253.

Amblyseius tunus McMurtry \& Moraes, 1989: 181; Feres \& Moraes, 1998: 126.

Neoseiulus tunus Moraes et al., 2000: 248; Chant \& McMurtry, 2003: 21.

Plantas em que foi encontrado: Cecropia sp., Heliotropium sp. e Lantana sp.

As espécies de fitoseídeos mais abundantes foram $E$. citrifolius e $N$. tunus, correspondendo a 189 (56\%) e 138 (41\%), respectivamente, do total de espécimes coletados (Tab. II).

Sobre Cecropia sp., foi observada a maior diversidade de fitoseídeos (5 espécies) e também a maior abundância, com 231 espécimes, dos quais 93 (40\%) pertenciam à espécie $E$. citrifolius e 135 (58\%) à N. tunus (Tab. II). Estes números correspondem á $50 \%$ de todos os E. citrifolius e $98 \%$ de todos os N. tunus coletados. Somadas, estas espécies foram encontradas em maior número na coleta realizada em julho (40\%) do que naquelas realizadas em abril (25\%), outubro (21\%) e janeiro $(14 \%)$.

Também sobre Cecropia sp. foi encontrado o maior número de fitoseídeos por amostra (29). Em seguida, as plantas que tiveram maior abundância de fitoseídeos por amostra foram: P. duncum (22), Guarea sp. (18) e A. conyzoides (12). Nenhum fitoseídeo foi encontrado sobre Cayaponia sp., a segunda espécie de planta mais amostrada neste trabalho, nem sobre Albizia hassleri (Chodat) Burkat, Hybanthus communis (A. St. Hil.) Taub., Indigofera sp. e Marsypianthes chamaedrys (Vahl) Kuntze.

\section{DISCUSSÃO}

Das seis espécies de fitoseídeos encontradas, cinco pertencem à subfamília Amblyseiinae e uma à subfamília Typhlodrominae. Este padrão de composição é compatível com aqueles observados em outros levantamentos conduzidos no Brasil sobre diferentes grupos de plantas (Denmark \& Muma 1973, Moraes et al. 1993, Feres \& Moraes 1998, Feres \& Nunes 2001, Gondim JR. \& Moraes 2001, Zacarias \& Moraes 2001, Ferla \& Moraes 2002b).

Os fitoseídeos encontrados no presente trabalho são muito comuns no Estado de São Paulo (Moraes et al. 2004). E. citrifolius tem sido verificado em um maior número de espécies de plantas que os outros fitoseídeos (Gravena et al. 1993, Feres \& Moraes 1998, Gondim Jr. \& Moraes 2001, Zacarias \& Moraes 2001, Feres et al. 2003, Moraes et al. 2004). A maior abundância e freqüência observada para E. citrifolius e N. tunus são compatíveis com os resultados obtidos por Feres \& Moraes (1998), que observaram estas espécies como os fitoseídeos mais abun-

Revista Brasileira de Zoologia 22 (1): 35-42, março 2005 
Tabela I. Espécies de plantas espontâneas visualmente mais abundantes, em diferentes épocas, em duas áreas de cultivo de seringueira no município de Olímpia, Estado de São Paulo.

\begin{tabular}{|c|c|c|}
\hline \multirow{2}{*}{ Época de coleta } & \multicolumn{2}{|c|}{ Espécies de plantas amostradas } \\
\hline & Área I & Área II \\
\hline \multirow{5}{*}{ Julho 2002} & Ageratum conyzoides $^{1}$ & Cayaponia sp. \\
\hline & Cecropia sp. ${ }^{2}$ & Cecropia sp. \\
\hline & Piper aduncum ${ }^{3}$ & Erechtites sp. ${ }^{13}$ \\
\hline & Solanum americanum ${ }^{4}$ & Marsypianthes chamaedrys ${ }^{14}$ \\
\hline & Sonchus sp. ${ }^{5}$ & Panicum maximum ${ }^{15}$ \\
\hline \multirow{5}{*}{ Outubro 2002} & Albizia hassleri ${ }^{6}$ & Cayaponia sp. \\
\hline & Cayaponia sp. ${ }^{7}$ & Cecropia sp. \\
\hline & Cecropia sp. & Eupatorium sp. ${ }^{16}$ \\
\hline & Hybanthus communis ${ }^{8}$ & Marsypianthes chamaedrys \\
\hline & Piper sp. ${ }^{9}$ & Panicum maximum \\
\hline \multirow{5}{*}{ Janeiro 2002} & Albizia hassleri & Capsicum sp. ${ }^{17}$ \\
\hline & Cayaponia sp. & Cecropia sp. \\
\hline & Cecropia sp. & Heliotropium sp. ${ }^{18}$ \\
\hline & Indigofera sp. ${ }^{10}$ & Panicum maximum \\
\hline & Piper sp. & Piper sp. \\
\hline \multirow{5}{*}{ Abril 2002} & Cecropia sp. & ?Amphilophium sp. ${ }^{19}$ \\
\hline & Guarea sp. ${ }^{11}$ & Astronium sp. ${ }^{20}$ \\
\hline & Lantana sp. ${ }^{12}$ & Cecropia sp. \\
\hline & Piper sp. & Heliotropium sp. \\
\hline & Solanum americanum & Lantana sp. \\
\hline
\end{tabular}

Anacardiaceae (20); Asteraceae (1, 5, 13, 16); Bignoniaceae (19); Boraginaceae (18); Cecropiaceae (2); Cucurbitaceae (7); Fabaceae (6, 10); Lamiaceae (14); Meliaceae (11); Piperaceae (3, 9); Poaceae (15); Solanaceae $(4,17)$; Verbenaceae (12); Violaceae (8).

dantes em um estudo conduzido em áreas de vegetação natural do oeste do Estado de São Paulo. A maior amplitude de substratos de E. citrifolius em relação a N. tunus pode estar relacionada ao hábito alimentar mais generalista de espécies do gênero Euseius que de Neoseiulus (McMurtry \& Croft 1997).

Segundo Noronha \& Moraes (2002), E. citrifolius é comumente encontrado em plantas de folhas lisas em várias regiões do Brasil. Esta foi a espécie mais freqüente em seringais localizados nos municípios paulistas de Barretos, Ibitinga, Macaubal (Feres 2000), Cedral, Pindorama e Taquaritinga (FEREs et al. 2002). A marcante presença deste fitoseídeo em Cecropia sp., que possui folhas bastante pilosas, sugere que plantas com esta característica também podem ser favoráveis à ocorrência desta espécie. Cecropia sp. é encontrada com grande facilidade nas entrelinhas das seringueiras. Trata-se de uma planta de fácil dispersão, sendo comumente encontrada em clareiras de matas, margem de estradas (LoNGino 1989) e nos estágios iniciais de recuperação de áreas perturbadas (Oliveira 2002).

As espécies G. annectens, I. zuluagai e N. tunus também são muito freqüentes no Estado de São Paulo, embora ocorram em menor diversidade de plantas (Denmark \& Muma 1973, Feres \& Moraes 1998, Gondim Jr. \& Moraes 2001, Zacarias \& Moraes
2001). Apesar de ter sido a segunda espécie mais abundante neste trabalho, $N$. tunus aparentemente não é um predador importante em seringueira, pois nunca foi encontrado sobre esta planta no Estado de São Paulo e apenas um indivíduo foi registrado no Estado do Mato Grosso sobre a mesma planta (Ferla \& Moraes 2002a). Dados da literatura sugerem que $I$. zuluagai possa ser um predador importante em seringueira. Esta espécie foi encontrada em números consideráveis em seringal circundado por plantação de citros em Taquaritinga, atingindo o pico populacional na mesma época que T. heveae, séria praga da seringueira (Feres et al. 2002). Entretanto, esta espécie não tem sido encontrada em cultivos de seringueiras em outras situações. Apesar de pertencer ao mesmo gênero da espécie mais abundante no presente trabalho e que também foi encontrada sobre a maior diversidade de plantas, E. concordis foi encontrado em número reduzido e em apenas uma planta. Assim como E. citrifolius e I. zuluagai, E. concordis é uma espécie muito comum e abundante em plantações de citros (SATo et al. 1994). Diferentemente do que se observa na região onde o presente trabalho foi conduzido, E. concordis parece ser o fitoseídeo mais freqüentemente encontrado em seringueiras no Estado do Mato Grosso (Feres 2000, Ferla \& Moraes 2002a). 
Tabela II. Fitoseídeos coletados sobre diferentes espécies de plantas em cultivos de seringueira no município de Olímpia, São Paulo, em julho e outubro de 2002 e janeiro e abril de 2003.

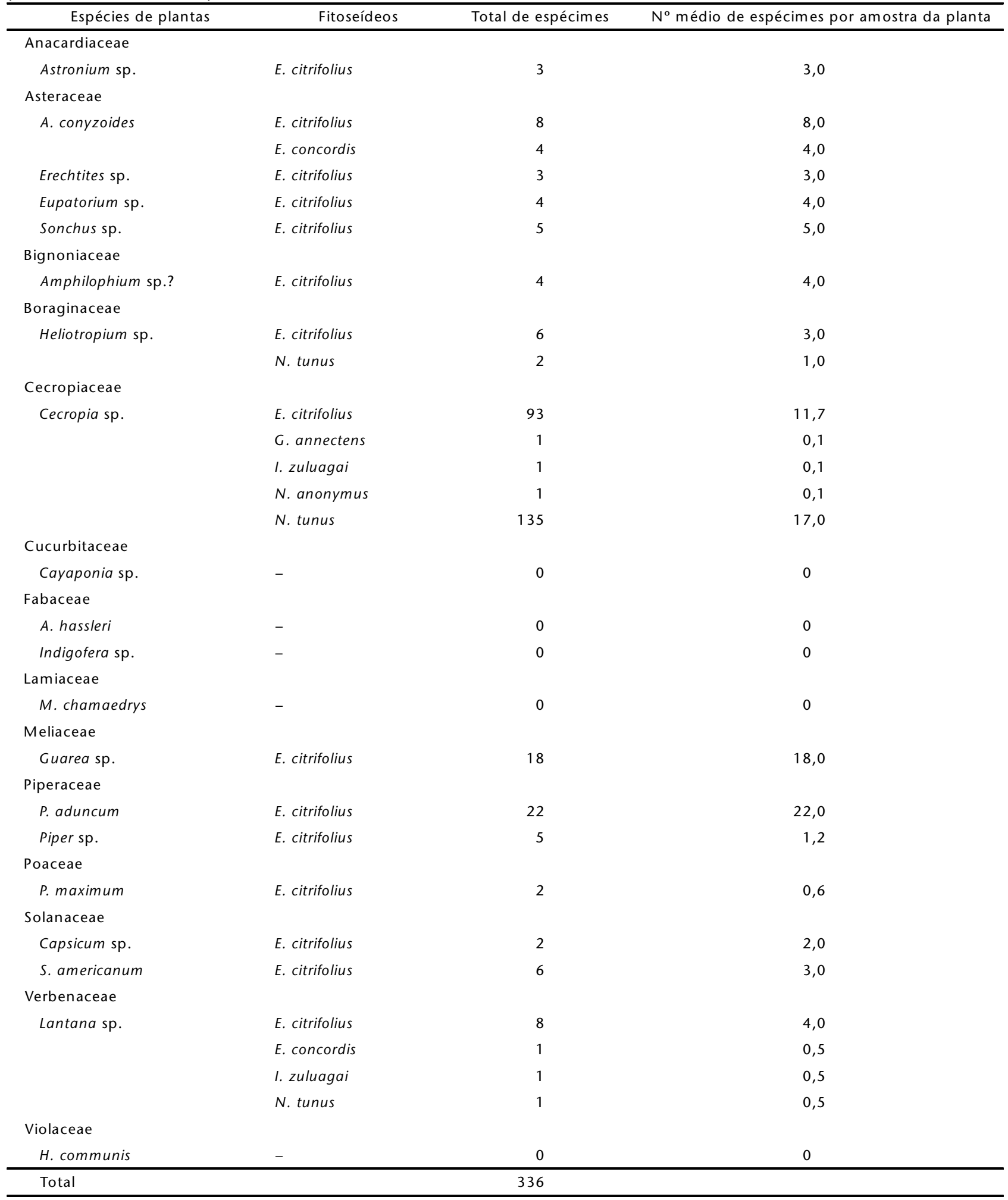


Além de São Paulo, os fitoseídeos coletados neste estudo também têm sido encontrados em vários outros estados do Brasil, assim como em outros países das Américas do Sul e Central. G. annectens e N. anonymus foram também registrados na América do Norte (Moraes et al. 2004). E. concordis foi ainda relatado na Iugoslávia e Portugal, mas é possível que os registros desta espécie na Europa correspondam na verdade a identificações errôneas (MoraEs et al. 2004).

Apesar de não identificados ao nível de espécie e não quantificados, ácaros fitófagos das famílias Eriophyidae, Tenuipalpidae e Tetranychidae foram freqüentemente observados sobre P. aduncum, Guarea sp. e A. conyzoides. Isto pode ter sido uma das causas de abundância relativamente grande de fitoseídeos nas amostras destas plantas. Mas como explicar a alta abundância de E. citrifolius e N. tunus em Cecropia sp., sendo que neste trabalho não foi observada a presença de flores (todas as plantas eram jovens) e ácaros de qualquer outra família sobre estas plantas? Uma possibilidade seria a presença de muitos tripes e pulgões, na maioria das amostras desta planta. Diversas espécies de fitoseídeos alimentam-se de tripes ou da substância açucarada produzida pelos pulgões (McMurTry et al. 1970). No presente trabalho, foi observado um exemplar de E. citrifolius predando uma espécie não identificada de tripes.

Há pelo menos duas possíveis explicações para a ausência de fitoseídeos em Cayaponia sp., A. hassleri, H. communis, Indigofera sp. e $M$. chamaedrys; a primeira delas poderia ser a falta de abrigo ou alimento aos predadores. A segunda possibilidade parece particularmente provável em relação a Cayaponia sp., que apresenta folhas pequenas e sem nervuras proeminentes. Entretanto, deve-se considerar que A. hassleri, $H$. communis, Indigofera sp. e M. chamaedrys foram coletadas apenas em uma ou duas ocasiões, reduzindo assim a chance de que predadores fossem coletados sobre elas. Por outro lado, a constatação de alguns poucos ácaros predadores sobre uma planta não significa que ela possa ser considerada como seu reservatório. Em P. maximum, por exemplo, foram encontrados apenas dois espécimes de fitoseídeos em três amostras da planta, sugerindo que estes pudessem estar por acaso sobre esta planta, durante o processo de dispersão.

Piper aduncum, Guarea sp. e A. conyzoides poderiam se tornar importantes em programas de manejo que envolvessem sua multiplicação, de forma semelhante ao que tem sido realizado em práticas envolvendo A. conyzoides em cultivos de citros (MAI et al. 1984?, Gravena et al. 1993, Liang et al. 1994, Mele \& Lenteren 2002). Cecropia sp. parece ser naturalmente mais importante que as outras plantas aqui estudadas, dada a freqüência com que é encontrada no seringal (planta perene) e também ao fato de que a maior abundância de E. citrifolius sobre esta planta tenha ocorrido em julho, época em que a desfolha das seringueiras é bastante pronunciada. Assim, Cecropia sp. poderia contribuir para que populações de $E$. citrifolius fossem mantidas nos plantios de seringueira nas épocas do ano em que a população deste predador sobre aquela é reduzida.

\section{AGRADECIMENTOS}

À Fundação de Amparo à Pesquisa do Estado de São Paulo (FAPESP), no âmbito do programa BIOTA/FAPESP - O Instituto Virtual da Biodiversidade (http://www.biota.org.br). À Plantações E. Michelin Ltda e Guaporé Pecuária S.A., pelo apoio financeiro. Ao Departamento de Zoologia e Botânica da UNESP de São José do Rio Preto, onde a pesquisa foi desenvolvida. À Profa Dra Neusa Taroda Ranga do Departamento de Zoologia e Botânica da UNESP de São José do Rio Preto, pela identificação das plantas analisadas neste trabalho. Aos produtores Gilmar Moretti e Miguel Francisco, que cederam suas propriedades para pesquisa de campo.

\section{REFERÊNCIAS BIBLIOGRÁFICAS}

Altieri, M.A.; E.N. Silva \& C.I. Nicholls. 2003. O papel da biodiversidade no manejo de pragas. Ribeirão Preto, Editora Holos, 226p.

Aponte, O. \& J.A. McMurtry. 1995. Revision of the genus Iphiseiodes De Leon (Acari: Phytoseiidae). International Journal of Acarology, West Bloomfield, 21 (3): 165-183.

Chant, D.A. 1959. Phytoseiid mites (Acarina: Phytoseiidae). Part I. Bionomics of seven species in southeastern England. Part II. A taxonomic review of the family Phytoseiidae, with descriptions of 38 new species. Canadian Entomologist, Ottawa, 91, (Supplement 12): 1-166.

Chant, D.A. \& E W. Baker. 1965. The Phytoseiidae (Acarina) of Central America. Memoirs of the Entomological Society of Canada, Ottawa, 41: 1-56.

Chant, D.A \& E. Yoshida-Shaul. 1984. A world review of the occidentalis species group in the genus Typhlodromus Scheuten (Acarina: Phytoseiidae). Canadian Journal of Zoology, Ottawa, 62: 1860-1871.

Chant, D.A \& J.A. McMurtry. 2003. A review of the subfamily Amblyseiinae Muma (Acari: Phytoseiidae): Part I. Neoseiulini new tribe. International Journal of Acarology, West Bloomfield, 29 (1): 3-46.

DeLeon, D. 1958. Four new Typhlodromus from southern Florida (Acarina: Phytoseiidae). Florida Entomologist, Gainesville, 40: 73-76.

Deleon, D. 1967. Some mites of the Caribbean Area. Lawrence, Kansas, Allen Press Inc., 66p.

Denmark, H.A. \& M.H. Muma. 1970. Some phytoseiid mites of Paraguay (Phytoseiidae: Acarina). Florida Entomologist, Gainesville, 53 (4): 219-227.

Denmark, H.A. \& M.H. Muma. 1972. Some Phytoseiidae of Colombia (Acarina: Phytoseiidae). Florida Entomologist, Gainesville, 55 (1): 19-29.

Denmark, H.A. \& M.H. Muma. 1973. Phytoseiid mites of Brazil (Acarina: Phytoseiidae). Revista Brasileira de Biologia, Rio de Janeiro, 33 (2): 235-276.

Emden, H.F. van. 1965. The role of uncultivated land in the biology of crop pests and beneficial insects. Scientific Horti-

Revista Brasileira de Zoologia 22 (1): 35-42, março 2005 
culture, Kent, 17: 121-136.

Feres, R.J.F. 2000. Levantamento e observações naturalísticas da acarofauna (Acari, Arachnida) de seringueiras cultivadas (Hevea spp., Euphorbiaceae) no Brasil. Revista Brasileira de Zoologia, Curitiba, 17 (1): 157-173.

Feres, R.J.F. \& G.J. De Moraes. 1998. Phytoseiid mites (Acari: Phytoseiidae) from woody areas in the State of São Paulo, Brazil. Systematic and Applied Acarology, London, 3: 125132.

Feres, R.J.F. \& M.A. Nunes. 2001. Ácaros (Acari, Arachnida) associados a euforbiáceas nativas em áreas de cultivo de seringueiras (Hevea brasiliensis Muell. Arg., Euphorbiaceae) na região noroeste do Estado de São Paulo, Brasil. Revista Brasileira de Zoologia, Curitiba, 18 (4): 1253-1264.

Feres, R.J.F.; D. De C. Rossa Feres; R.D. Daud \& R.S. Santos. 2002. Diversidade de ácaros (Acari, Arachnida) em seringueiras (Hevea brasiliensis Muell. Arg., Euphorbiaceae) na região noroeste do Estado de São Paulo, Brasil. Revista Brasileira de Zoologia, Curitiba, 19 (1): 137-144.

Feres, R.J.F.; M.R. Bellini \& D. De C. Rossa-Feres. 2003. Ocorrência e diversidade de ácaros (Acari, Arachnida) associados a Tabebuia roseo-alba (Ridl.) Sand (Bignoniaceae), no município de São José do Rio Preto, São Paulo, Brasil. Revista Brasileira de Zoologia, Curitiba, 20 (3): 373-378.

Ferla, N.J. \& G.J. de Moraes. 2002a. Ácaros (Arachnida, Acari) da seringueira (Hevea brasiliensis Muell. Arg.) no Estado do Mato Grosso, Brasil. Revista Brasileira de Zoologia, Curitiba, 19 (3): 867-888.

Ferla, N.J. \& G.J. De Moraes. 2002b. Ácaros predadores (Acari) em plantas nativas e cultivadas do Estado do Rio Grande do Sul, Brasil. Revista Brasileira de Zoologia, Curitiba, 19 (4): 1011-1031.

Flechtmann, C.H.W. 1975. Elementos de Acarologia. São Paulo, Livraria Nobel S.A., 344p.

Gerson, U.; R.L. Smiley \& R. Ochoa. 2003. Mites (Acari) in Biological Control. Boston, Blackwell Science, 539p.

Gondim JR., M.G.C. \& G.J. DE Moraes. 2001. Phytoseiid mites (Acari: Phytoseiidae) associated with palm trees (Arecaceae) in Brazil. Systematic and Applied Acarology, London, 6: 65-94.

Gravena, S.; A. Coletti \& P.T. Yamamoto. 1993. Influence of green cover with Ageratum conyzoides and Eupatorium pauciflorum on predatory and phytophagous mites in citrus. Bulletin International Organization for Biological and Integrated Control of Noxious Animals and Plants: West Palearctic Regional Section, Avignon, 16 (7): 104-114.

Ismail, B.S.; A. Tasrif; S.S. Sastroutomo \& A. Latiff. 1995. Weed seed populations in rubber and oil palm plantations with legume cover crops. Plant Protection Quarterly, Victoria, 10 (1): 20-23.

LAM, C.H.; J.K. Lim \& B. JANTAN. 1993. Comparative studies of a paraquat mixture and glyphosate and/or its mixtures on weed succession in plantation crops. Planter, Kuala Lumpur,
69 (812): 525-535.

Liang, W.G.; W. HuI \& W.K. LeE. 1994. Influence of citrus orchard ground cover plants on arthropod communities in China: A review. Agriculture Ecosystems and Environment, Amsterdam, 50 (1): 29-37.

LongINo, J.T. 1989. Geographic variation and community structure in na ant-plant mutualism: Azteca and Cecropia in Costa Rica. Biotropica, Washington, 21 (2): 126-132.

MaI, X.H.; S.X. LI; J.J. Xong; D.S. Zheng; M.D. Huang \& T.J. SI. 1984. Studies on the relationship between the ecological factors (presence of Ageratum conyzoides) and population of predaceous mite and its utilization for the control of citrus red mite. Acta Phytophylacica Sinica, Peking, 11 (1): 2934.

McMurtry, J.A. 1983. Phytoseiid mites from Guatemala, with descriptions of two new species and redefinitions of the genera Euseius, Typhloseiopsis, and the Typhlodromus occidentalis species group (Acari: Mesostigmata). International Journal of Entomology, Honolulu, 25 (4): 249-272.

McMurtry, J.A. \& B.A. Croft. 1997. Life-styles of phytoseiid mites and their roles in biological control. Annual Review of Entomology, Palo Alto, 42: 291-321.

McMurtry, J.A. \& G.J. de Moraes. 1989. Some phytoseiid mites from Peru with descriptions of four new species (Acari: Phytoseiidae). International Journal of Acarology, West Bloomfield, 15 (3): 179-188.

McMurtry, J.A.; C.B. Huffaker \& M. van de Vrie. 1970. Ecology of tetranychid mites and their natural enemies: a review. I. Tetranychid enemies: their biological characters and the impact of spray practices. Hilgardia, Berkeley, 40 (11): 331390.

Mele, P. van \& J.C. van Lenteren. 2002. Survey of current crop management practices in a mixed-ricefield landscape, Mekong Delta, Vietnam: Potential of habitat manipulation for improved control of citrus leafminer and citrus red mite. Agriculture Ecosystems and Environment, Amsterdam, 88 (1): 35-48.

Moraes, G. J. de \& J. A. McMurtry. 1983. Phytoseiid mites (Acarina) of northeastern Brazil with descriptions of four new species. International Journal of Acarology, West Bloomfield, 9 (3): 131-148.

Moraes, G.J. de \& N.C. Mesa. 1988. Mites of the family Phytoseiidae (Acari) in Colombia, with descriptions of three new species. International Journal of Acarology, West Bloomfield, 14 (2): 71-88.

Moraes, G.J. de; H.A. Denmark \& J.M. Guerrero. 1982. Phytoseiid mites of Colombia (Acarina: Phytoseiidae). International Journal of Acarology, West Bloomfield, 8 (1): 15-22.

Moraes G.J. De; J.A. de Alencar; J.L.S. de Lima, J.S. Yaninek \& I. Delalibera JR. 1993. Alternative plant habitats for common phytoseiid predators of the cassava green mite (Acari: Phytoseiidae, Tetranychidae) in northeast Brazil. Experimental and Applied Acarology, Amsterdam, 17: 77-90. 
Moraes, G.J. de \& S. Kreiter \& A.C. Lofego. 2000. Plant mites (Acari) of the French Antilles. 3. Phytoseiidae (Gamasida). Acarologia, Paris, 15 (3): 237-264.

Moraes, G.J. de; J A. McMurtry; H A. Denmark \& C B. Campos. 2004. A revised catalog of the mite family Phytoseiidae. Zootaxa, Auckland, 434: 1-494.

Muma, M.H. 1961. Subfamilies, genera and species of Phytoseiidae (Acarina: Mesostigmata). Bulletin Florida State Museum, Biological Science, Gainesville, 5 (7): 267-302.

Muma, M.H. 1963. The genus Galendromus Muma, 1961 (Acarina: Phytoseiidae). Florida Entomologist, Gainesville, Supplement, 1: 15-41.

Noronha, A.C. DA S. \& G.J. De Moraes. 2002. Variações morfológicas intra e interpopulacionais de Euseius citrifolius Denmark \& Muma e Euseius concordis (Chant) (Acari, Phytoseiidae). Revista Brasileira de Zoologia, Curitiba, 19 (4): 1111-1122.

OliveirA, R.R. DE. 2002. Ação antrópica e resultantes sobre a estrutura e composição da Mata Atlântica na Ilha Grande, RJ. Rodriguésia, Rio de Janeiro, 53 (82): 33-58.

Pontier, K.J.B.; G.J. De Moraes \& S. Kreiter. 2001. Biology of Tenuipalpus heveae (Acari, Tenuipalpidae) on rubber tree leaves. Acarologia, Paris, 41 (4): 423-427.

Sato, M.E.; A. Raga; L.C. Cerávolo; A.C. Rossi \& M.R. Potenza. 1994. Ácaros predadores em pomar cítrico de Presidente Prudente, Estado de São Paulo. Anais da Sociedade Entomológica do Brasil, Londrina, 23 (3): 435-441.

Schicha, E. \& M. Elshafie. 1980. Four new species of phytoseiid mites from Australia, and three species from America redescribed (Acari: Phytoseiidae). Journal of the Australian Entomological Society, Brisbane, 19: 27-36.

Thresh, J.M. 1981. Pests, pathogens, and vegetation: the role of weeds and wild plants in the ecology of crop pests and diseases. Boston, Pitman Advanced Publishing Program, $517 \mathrm{p}$.

VieirA, M.R. \& E.C. Gomes. 1999. Sintomas, desfolhamento e controle de Calacarus heveae Feres, 1992 (Acari, Eriophyidae) em seringueira (Hevea brasiliensis Muell. Arg.). Cultura Agronômica, Ilha Solteira, 8 (1): 39-52.

Zacarias, M.S. \& G.J. De Moraes. 2001. Phytoseiid mites (Acari) associated with rubber trees and other euphorbiaceous plants in southeastern Brazil. Neotropical Entomology, Londrina, 30 (4): 579-586.

Recebido em 13.IV.2004; aceito em 05.I.2005.

Revista Brasileira de Zoologia 22 (1): 35-42, março 2005 